\title{
Pigmentación inusual del paladar asociada a imatinib. Reporte de caso clínico
}

\section{Unusual pigmentation of palate associated to imatinib. A case report}

\section{López-Labady J*, Villarroel-Dorrego M**, Bascones Martínez A***}

\section{RESUMEN}

Existen diversos factores que pueden inducir pigmentaciones en la mucosa bucal, entre ellos algunos fármacos tienen la capacidad de estimular la producción de melanina en el epitelio oral. Recientemente se ha demostrado que el mesilato de imatinib (Glivec ${ }^{\circledR}$ ) usado como droga antineoplásica es capaz de producir como efecto adverso pigmentación de la mucosa bucal muy especialmente la del paladar. Se reporta el caso de una paciente de 56 años de edad bajo terapia con imatinib desde hace 8 años por padecer de leucemia mieloide crónica. Al examen clínico se observó una hiperpigmentación color azul grisácea en la totalidad del paladar óseo que al estudio histopatológico mostró acúmulo de melanina principalmente en el corion. La paciente se ha mantenido con la lesión en paladar bajo observación, sin cambios clínicos ni histopatológicos. Para establecer la asociación de este fármaco con la pigmentación en la mucosa bucal es necesario descartar otros factores inductores de melanosis además de considerar las características clínicas como coloración y ubicación de la lesión.

Palabras clave: Mesilato de imatinib, hiperpigmentación, mucosa palatina.

\section{SUMMARY}

There are several factors related to oral mucosa pigmentations, among them; some drugs may be able to induce melanin production in oral epithelia. Recently, it has been demonstrated that imatinib metylate (Glivec $($ )), used to stop tumor growth, may cause oral pigmentation especially on the hard palate. A case is reported regarding a 56 year old female under imatinib treated since 8 years for chronic myeloid leukemia. Oral evaluation showed a diffuse blue/grey hyperpigmentation covering completely hard palate. Histopathologycal analysis revealed melanin accumulation in corion. Patient is under clinical surveillance, with no clinical changes. In order to establish the association between imatinib and oral pigmentation is necessary to discard other factors that may induce melanin production.

Key words: Imatinib mesylate, oral pigmentation, palatal mucosa.

Fecha de recepción: 13 de abril de 2013.

Aceptado para publicación: 15 de mayo de 2013.

López-Labady J, Villarroel-Dorrego M, Bascones Martínez A. Pigmentación inusual del paladar asociada a imatinib. Reporte de caso clínico. Av. Odontoestomatol 2013; 29 (6): 309-314.

* Profesora Asociada. Cátedra de Anatomía Patológica. Facultad de Odontología.Universidad Central de Venezuela, Caracas. Venezuela.

** Profesora Asociada del Instituto de Investigaciones Odontológicas. Universidad Central de Venezuela.

*** Caterático de Medicina Bucal y Periodoncia e Implantes. Universidad Complutense de Madrid, Madrid. España. 


\section{INTRODUCCIÓN}

La mucosa bucal con relativa frecuencia muestra pigmentaciones relacionadas con la producción excesiva de melanina, las cuales pueden ser difusas o focalizadas (1). Pueden estar asociadas a pigmentos endógenos como la melanina o de origen exógeno como los tatuajes siendo el de amalgama el más frecuente. Entre las pigmentaciones melánicas focalizadas de la mucosa bucal se encuentran la mácula melánica y los nevus como los más comunes, y entre las difusas tenemos las pigmentaciones asociadas al origen étnico, a enfermedades sistémicas, tabaquismo y fármacos (2).

Son múltiples los fármacos de los cuales existe evidencia de su capacidad de inducir pigmentaciones en mucosa bucal, como por ejemplo la cloroquina, la ciclofosfamida, el ketoconazol, la clorhexidina, anticonceptivos orales, la tetraciclina y más recientemente reportado en la literatura el mesilato de imatinib entre otros $(3,4)$.

El mesilato de imatinib (Glivec/Gleevec $®$ ) es un fármaco inhibidor específico de la quinasa tirosina, que recientemente ha sido aprobado como tratamiento de primera línea para pacientes con diagnóstico de leucemia mieloide crónica que no sean candidatos para trasplante de médula ósea alogénico y también para otras patologías como síndromes mielodisplásicos o mieloproliferativos, leucemia eosinofílica crónica y pacientes con tumores del estroma gastrointestinal (5). Su modo de acción lo logra al unirse a los sitios activos de la proteína quinasa tirosina previniendo su actividad enzimática, corrigiendo así los síntomas de la enfermedad $(2,6)$.

Se describen efectos secundarios como náuseas, diarrea, edema periorbital, mielosupresión y dolor musculoesquelético entre otros. Las complicaciones cutáneas son diversas siendo la más frecuente la hipopigmentación y así lo demuestra el estudio de Arora et al (5) con $41 \%$ de los casos estudiados, sin embargo en ciertas condiciones se puede ver hiperpigmentación de piel y mucosas (7). Existen nuevos reportes en la literatura de pigmentación difusa del paladar óseo en pacientes bajo este tratamiento en donde la piel podría también estar afectada de forma concomitante (2).

\section{PRESENTACIÓN DE CASO CLÍNICO}

Se trata de paciente caucásica de 56 años de edad, quien acude a consulta referida por su médico oncólogo por presentar lesión macular en paladar óseo. Al interrogatorio refiere diagnóstico de leucemia mieloide crónica desde hace 10 años motivo por el cual está medicada únicamente con $400 \mathrm{mg}$ diarios de mesilato de imatinib (Glivec ${ }^{\circledR}$ ) desde hace 8 años. Niega tabaquismo.

Al examen clínico intrabucal se observó una mácula azul grisácea no dolorosa limitada al paladar óseo que presentó bordes irregulares y difusos de la cual la paciente no puede dar datos evolutivos (Figura 1).

Al examen clínico facial se observó la presencia de máculas grisáceas distribuidas de manera bilateral en la zona temporal (Figuras 2 y 3 ).

Se decide indicar exámenes hematológicos y se establece interconsulta con su médico tratante para tomar biopsia incisional de la lesión (Figura 4). El estudio histopatológico describe acúmulo citoplasmático de melanina (Figuras 5 y 6). Se presume que el medicamento es el elemento inductor de la lesión ya que se descartó cualquier otro factor desencadenante de pigmentación en mucosa bucal o piel.

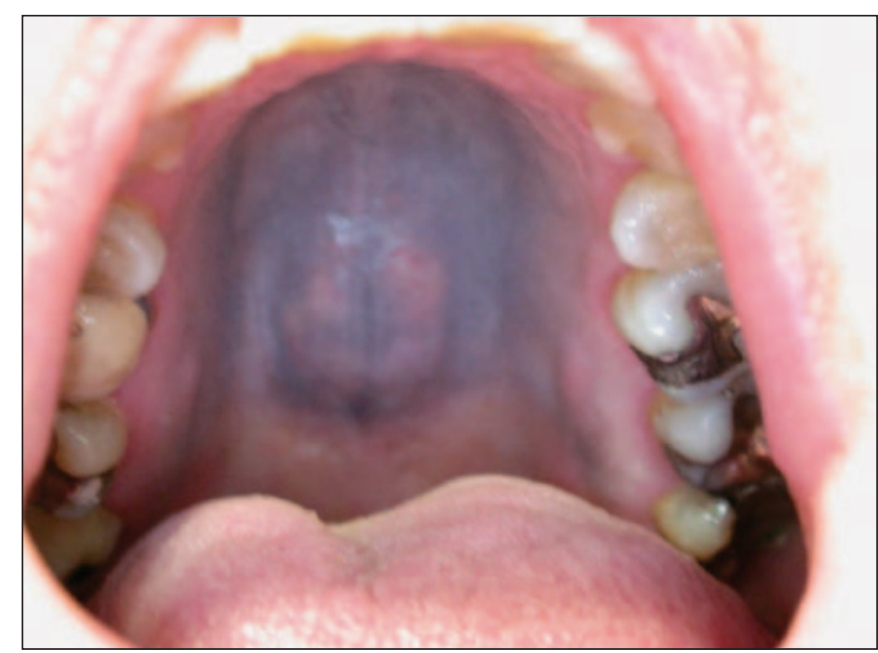

Fig. 1. Lesión macular azul grisácea que ocupa la totalidad de la bóveda palatina. 

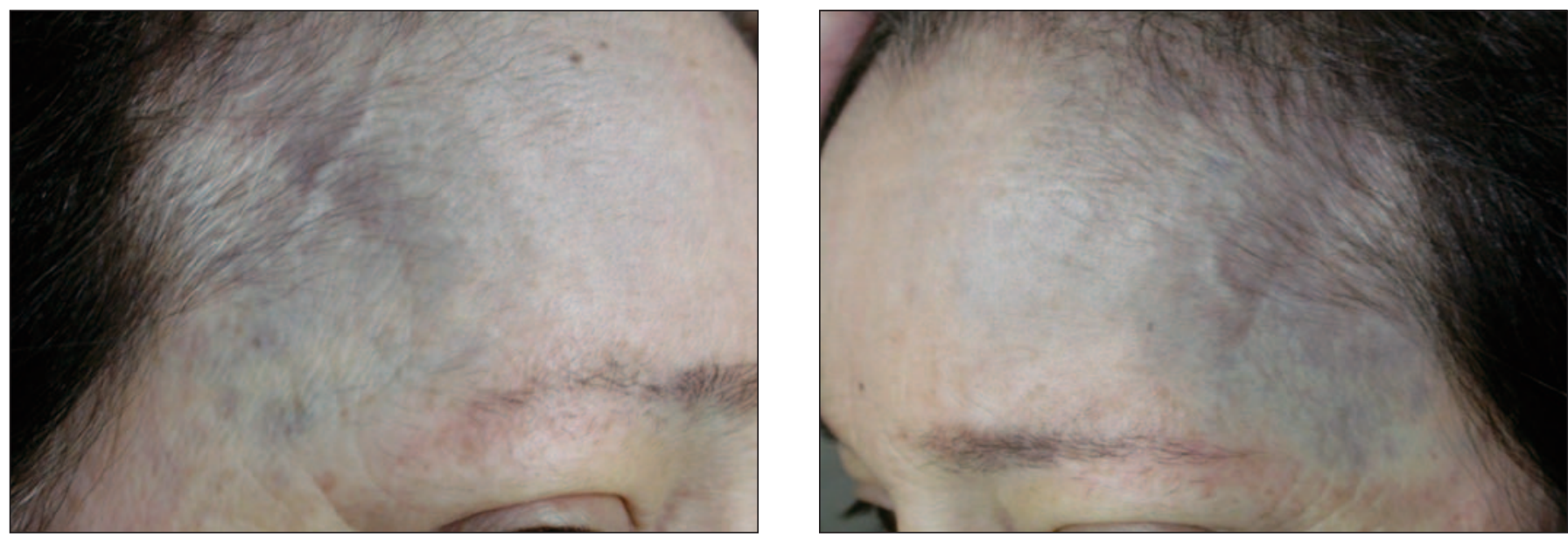

Figs. 2 y 3. Lesiones maculares grisáceas y difusas cutáneas ubicadas bilateralmente en zona temporal.

\section{DISCUSIÓN}

El mesilato de imatinib es una droga de primera elección para el tratamiento de la leucemia mieloide crónica, cuyos efectos adversos son bien conocidos y entre ellos se destacan los cutáneos muy especialmente la hipopigmentación y más recientemente se reconoce su capacidad de inducir hiperpigmentación tanto en piel como en mucosa bucal $(2,5)$.

La paciente reportada en este estudio mostró hiperpigmentación de la mucosa bucal asociada a tratamiento prolongado con imatinib. Fue el paladar óseo el área afectada y pareciera ser la zona predilecta de

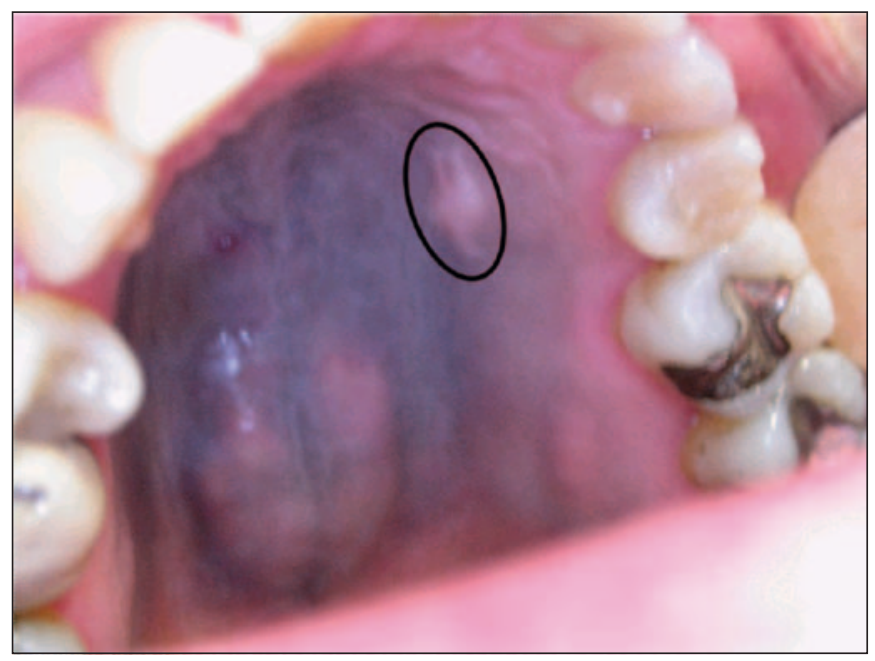

Fig. 4. Zona identificada de la toma de biopsia.
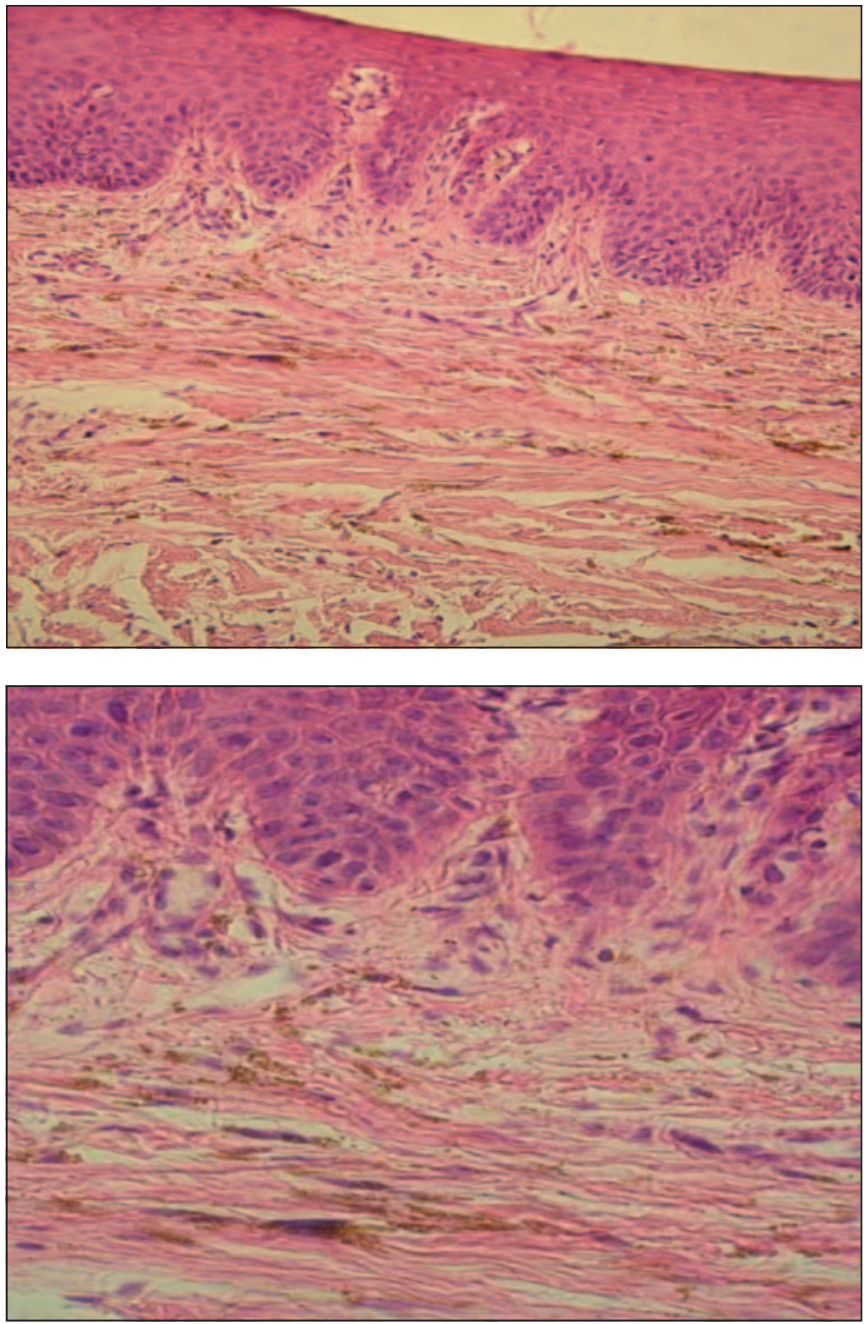

Figs. 5 y 6. Microfotografías del estudio histopatológico que muestra el acúmulo citoplasmático de melanina, fundamentalmente en el corion. 
aparición como consecuencia de la terapia pues también ha sido reportada por la mayoría de los autores (Tabla 1), siendo la única excepción el caso referido por Mcpherson et al (8) quienes solo la observaron en encía.

Se desconoce la causa de la predilección anatómica dentro de la cavidad bucal, pero es relevante el hecho de que se trata de mucosa adherida a hueso la que tiene mayor disposición a ser afectada, en este sentido debemos recordar que la encía y la mucosa palatina están asociadas al trauma inducido por la alimentación y éste muy bien podría ser un factor condicionante en la preferencia de ubicación.

También se pudo observar alteraciones de pigmentación en la piel, a diferencia de la mayoría de los casos bucales reportados hasta la fecha donde apreciaron alteraciones pigmentarias únicamente en paladar y encía. Sólo Resende et al (3) también reportó hiperpigmentación en ambos lóbulos de la oreja. La mayoría de los reportes en la literatura de efectos del

\section{TABLA 1. CASOS REPORTADOS EN LA LITERATURA DE PIGMENTACIÓN MUCO-CUTÁNEA ASOCIADA A IMATINIB}

\begin{tabular}{|c|c|c|c|c|c|}
\hline Autores & Enfermedad & Género & Edad & $\begin{array}{l}\text { Tiempo y dosis } \\
\text { de tratamiento }\end{array}$ & Localización \\
\hline $\begin{array}{l}\text { McPherson } \\
\text { et al ( } 8) \text { ( } 1 \text { caso) }\end{array}$ & LMC & Masculino & 59 años & 6 años & $\begin{array}{c}\text { Encía superior } \\
\text { y uñas }\end{array}$ \\
\hline $\begin{array}{l}\text { Lewis }(11) \\
(1 \text { caso) }\end{array}$ & LMC & Masculino & 70 años & 3 años & $\mathrm{PO}$ \\
\hline $\begin{array}{l}\text { Resende et al ( } 3 \text { ) } \\
\text { ( } 1 \text { caso) }\end{array}$ & LMC & Masculino & 38 años & 5 años & $\begin{array}{c}\text { PO } \\
\text { Ambos lóbulos } \\
\text { de la oreja y lado } \\
\text { izq. de la nariz }\end{array}$ \\
\hline $\begin{array}{l}\text { Mattson et al (1) } \\
(3 \text { casos) }\end{array}$ & $\begin{array}{l}\text { 1. Leiomioblastoma } \\
\text { 2. LMC } \\
\text { 3. LMC }\end{array}$ & $\begin{array}{l}\text { Femenino } \\
\text { Femenino } \\
\text { Femenino }\end{array}$ & $\begin{array}{l}66 \text { años } \\
66 \text { años } \\
64 \text { años }\end{array}$ & $\begin{array}{l}400 \text { mg/día x } 8 \text { años } \\
400 \text { mg/día x } 6 \text { años } \\
400 \text { mg/día x } 8 \text { años }\end{array}$ & $\begin{array}{l}\mathrm{PO} \\
\mathrm{PO} \\
\mathrm{PO}\end{array}$ \\
\hline $\begin{array}{l}\text { Wong et al (12) } \\
\text { (1 caso) }\end{array}$ & LMC & Femenino & 43 años & No se especifica & $\mathrm{PO}$ \\
\hline $\begin{array}{l}\text { Li et al }(2) \\
(3 \text { casos) }\end{array}$ & $\begin{array}{l}\text { 1. LMC } \\
\text { 2.LMC } \\
\text { 3. Fibromatosis } \\
\text { pélvica }\end{array}$ & $\begin{array}{l}\text { Masculino } \\
\text { Masculino } \\
\text { Femenino }\end{array}$ & $\begin{array}{l}64 \text { años } \\
53 \text { años } \\
29 \text { años }\end{array}$ & $\begin{array}{l}400 \text { mg/día x } 4 \text { años } \\
400 \text { mg/día x } 10 \text { años } \\
400 \text { mg/día x } 4 \text { años }\end{array}$ & $\begin{array}{l}\mathrm{PO} \\
\mathrm{PO} \\
\mathrm{PO}\end{array}$ \\
\hline $\begin{array}{l}\text { Steele et al (13) } \\
\text { (1 caso) }\end{array}$ & LMC & Masculino & 48 años & 400 mg/día x 9 años & $\begin{array}{l}\text { PO y uñas } \\
\text { sólo de los pies }\end{array}$ \\
\hline $\begin{array}{l}\text { López-Labady } \\
\text { et al ( } 1 \text { caso) }\end{array}$ & LMC & Femenino & 56 años & 400 mg/día x 8 años & $\begin{array}{c}\text { PO y piel } \\
\text { Zona temporal }\end{array}$ \\
\hline
\end{tabular}

LMC: Leucemia Mieloide Crónica. PO: Paladar Óseo 
imatinib, desde el punto de vista cutáneo, son descritos como hipopigmentación de la piel (9), de hecho es considerado unos de los efectos adversos más comunes del medicamento, al punto en que se ha convertido en una terapia de uso frecuente para la despigmentación de la piel normal en pacientes con vitíligo (10).

Desde el punto de vista clínico destaca la coloración de la pigmentación pues mostraba una tonalidad azul grisácea, característica que la diferencia de otras condiciones que bien podrían ser diagnósticos diferenciales relevantes como pigmentaciones raciales fisiológicas, melanosis por tabaquismo, máculas melánicas, enfermedad de Addison, síndrome de Peutz-Jeghers e incluso de un melanoma, pues se trata de condiciones que usualmente se muestran como pigmentaciones que varían de marrones parduzcas a negras, además de otros elementos claramente asociados como etnia, tabaco, condiciones genéticas y predilecciones anatómicas entre otras.

Por su lado lesiones como el nevus azul y los tatuajes de amalgama o de tinta, que podrían presentar una coloración similar a la observada en nuestro paciente, con el tamaño marcan la diferencia pues se trata de condiciones usualmente de pequeñas dimensiones que contrastan con una lesión que prácticamente ocupa la totalidad del paladar óseo (3). Otras patologías como hemocromatosis y beta-talasemia, que se asocian a pigmentación de la bóveda palatina (11), también fueron descartadas en nuestra paciente, al igual que el consumo de algún otro fármaco reportado en la literatura como inductor de hiperpigmentación mucosa como hidroxiúrea o antimaláricos entre otros.

Esta patología se caracteriza por no ser dolorosa, de hecho podría pasar desapercibida tanto por el clínico como por el paciente, por esta razón no requiere de tratamiento e incluso por su ubicación tampoco acarrea problemas estéticos (2), sin embargo es importante considerar evaluaciones bucales periódicas en aquellos pacientes bajo terapia con imatinib y de esta manera poder llevar un seguimiento adecuado y así permitir identificar cualquier expresión de la medicación.

Sus características clínicas como color, distribución y localización más la ausencia de síntomas le brindan al clínico claros indicios como para presumir que se trata de un efecto adverso en presencia de terapia farmacológica con imatinib, sobre todo porque los reportes en la literatura son consecuentes con estos rasgos, pero es la biopsia el elemento determinante que permitirá dar respuesta certera al paciente sobre su afección y a quien definitivamente por su condición médica es necesario descartar otras entidades que podrían representar un riesgo para su salud.

\section{CONCLUSIONES}

El presente reporte describe la capacidad de imatinib de inducir hiperpigementación de piel y mucosas como efecto secundario y la presencia de estas lesiones pareciera relacionarse a tratamiento prolongado con el medicamento. La pigmentación por el medicamento fue observada en el paladar óseo y podría ser una manifestación única o estar asociada también a alteraciones en la piel. Descartar patologías o factores capaces de inducir hiperpigmentación de la mucosa bucal debe ser parte vital del proceso para establecer el diagnóstico definitivo.

\section{BIBLIOGRAFÍA}

1. Mattsson U, Halbritter S, Mörner Serikoff E, Christerson L, Warfvinge G. Oral pigmentation in the hard palate associated with imatinib mesylate therapy: a report of three cases. Oral Surg Oral Med Oral Pathol Oral Radiol Endod. 2011;111(5): e12-6.

2. Li CC, Malik SM, Blaeser BF, Dehni WJ, Kabani SP, Boyle N, Toner M, Woo SB. Mucosal pigmentation caused by imatinib: report of three cases. Head Neck Pathol. 2012;6(2):290-5.

3. Resende RG, Teixeira RG, Vasconcelos FD, Silva ME, Abreu MH, Gomez RS. Imatinib-associated hyperpigmentation of the palate in post-HSCT patient. J Craniomaxillofac Surg. 2011;40:140-3.

4. Torres-Pereira CC. Are we ready to blame imatinib for palatal pigmentation? Oral Surg Oral Med Oral Pathol Oral Radiol Endod. 2011;112(3):287. 
5. Arora B, Kumar L, Sharma A, Wadhwa J, Kochupillai V. Pigmentary changes in chronic myeloid leukemia patients treated with imatinib mesylate. Ann Oncol. 2004;15(2):358-9.

6. Basso FG, Boer CC, Corrêa ME, Torrezan M, Cintra ML, de Magalhães MH, da Silva Santos P, de Souza CA. Skin and oral lesions associated to imatinib mesylate therapy. Support Care Cancer. 2009;17(4):465-8.

7. Legros L, Cassuto JP, Ortonne JP. Imatinib mesylate (Glivec): a systemic depigmenting agent for extensive vitiligo? $\mathrm{Br}$ J Dermatol. 2005;153 (3):691-2.

8. Mcpherson T, Sherman V, Turner R. Imatinibassociated hyperpigmentation, a side effect that should be recognized. J Eur Acad Dermatol Venereol. 2009; 23(1):82-3.

9. Aleem A. Hypopigmentation of the skin due to imatinib mesylate in patients with chronic myeloid leukemia. Hematol Oncol Stem Cell Ther. 2009; 2(2):358-61.

10. AlGhamdi KM, Kumar A. Depigmentation therapies for normal skin in vitiligo universalis. $\mathrm{J}$
Eur Acad Dermatol Venereol. 2011;25(7):74957.

11. Lewis DM. Diffuse pigmentation of the palate. $\mathrm{J}$ Okla Dent Assoc. 2009;100(8):24-5.

12. Wong M, Sade S, Gilbert M, Klieb H. Oral melanosis after tyrosine kinase inhibition with Imatinib for chronic myelogenous leukemia: Report of a case and review of the literature. Dermatol Online J. 2011;17(5):4.

13. Steele JC, Triantafyllou A, Rajlawat BP, Field EA. Oral mucosal hyperpigmentation and horizontal melanonychia caused by imatinib. Clin Exp Dermatol. 2012;37(4):432-3.

\section{CORRESPONDENCIA}

Dra. Jeaneth López Labady

Cátedra de Anatomía Patológica.

Facultad de Odontología, piso 8.

Universidad Central de Venezuela.

Los Chaguaramos 1060

Caracas. Venezuela.

Correo electrónico: jeanethlopez2306@hotmail.com 